\title{
Correction to: The SRCIN1/p140Cap adaptor protein negatively regulates the aggressiveness of neuroblastoma
}

\author{
Silvia Grasso ${ }^{1}$ - Davide Cangelosi ${ }^{2} \cdot$ Jennifer Chapelle ${ }^{1} \cdot$ Melissa Alzona $^{1} \cdot$ Giorgia Centonze $^{1}$. \\ Alessia Lamolinara $\mathbb{1}^{3} \cdot$ Vincenzo Salemme $^{1} \cdot$ Costanza Angelini $^{1} \cdot$ Alessandro Morellato $^{1} \cdot$ Andrea Saglietto $^{4}$. \\ Federico Tommaso Bianchi ${ }^{1,5}$. Sara Cabodi ${ }^{1}$ - Iris Chiara Salaroglio ${ }^{1,6}$. Federica Fusella ${ }^{1}$. Marzia Ognibene ${ }^{7}$. \\ Manuela lezzi $\mathbb{D}^{3} \cdot$ Annalisa Pezzolo $\mathbb{D}^{7} \cdot$ Valeria Poli $\mathbb{D}^{1} \cdot$ Ferdinando Di Cunto $\mathbb{D}^{5}$ - Alessandra Eva ${ }^{2}$. \\ Chiara Riganti $\mathbb{1}^{6} \cdot$ Luigi Varesio $^{2} \cdot$ Emilia Turco $^{1} \cdot$ Paola Defilippi $\mathbb{B}^{1}$
}

Published online: 5 September 2019

(c) The Author(s), under exclusive licence to ADMC Associazione Differenziamento e Morte Cellulare 2019

\section{Correction to: Cell Death \& Differentiation}

https://doi.org/10.1038/s41418-019-0386-6
This article was originally published under a standard license to Publish, but has now been made available under a CC BY license. The PDF and HTML versions of the paper have been modified accordingly.
These authors contributed equally: Silvia Grasso, Davide Cangelosi, Jennifer Chapelle, Melissa Alzona

These authors contributed equally to this work as co-last authors: Emilia Turco, Paola Defilippi

\section{Edited by G. Del Sal}

Luigi Varesio prematurely passed away in December 2017. The colleagues who had the privilege to collaborate with him dedicate this manuscript to his memory.

Paola Defilippi

paola.defilippi@unito.it

1 Department of Molecular Biotechnology and Health Sciences, University of Torino, 10126 Torino, Italy

2 Laboratory of Molecular Biology, Giannina Gaslini Institute, 16147 Genova, Italy

3 Department of Medicine and Aging Science, Center of Excellence on Aging and Translational Medicine (CeSi-Met), G. D'Annunzio University, Chieti-Pescara, Italy
4 Cardiology Division, Department of Medical Sciences, University of Torino, 10126 Torino, Italy

5 Neuroscience Institute Cavalieri Ottolenghi, Regione Gonzole 10, 10043 Orbassano (TO), Italy

6 Department of Oncology, University of Torino, 10126 Torino, Italy

7 Laboratorio Cellule Staminali Post Natali e Terapie Cellulari, Istituto Giannina Gaslini, Genova, Italy 